Revista Brasileira de Meteorologia, v.25, n.3, 311 - 323, 2010

\title{
MÉTODO OBJETIVO DE IDENTIFICAÇÃO DOS VÓRTICES CICLÔNICOS DE ALTOS NÍVEIS NA REGIÃO TROPICAL SUL: VALIDAÇÃO
}

\author{
MICHELYNE DUARTE LEAL COUTINHO, MANOEL ALONSO GAN, VADLAMUDI \\ BRAHMANANDA RAO
}

\author{
Centro de Previsão de Tempo e Estudo Climáticos, Instituto Nacional de Pesquisas Espaciais \\ (CPTEC/INPE), São José dos Campos, SP
}

michelyne.coutinho@cptec.inpe.br,manoel.gan@cptec.inpe.br, vbrao@cptec.inpe.br

\author{
Recebido Junho 2009 - Aceito Março 2010 \\ RESUMO
}

\begin{abstract}
Neste estudo foi desenvolvido um Método Objetivo (MO) para identificar Vórtices Ciclônicos de Altos Níveis (VCANs) na região Tropical Sul, baseado na vorticidade relativa e no escoamento horizontal do vento ao redor do centro dos sistemas. O MO foi elaborado usando dados de reanálises do National Centers for Environmental Prediction - National Centers for Atmospheric Research (NCEP-NCAR) e validado, para o período de cinco anos (2002 a 2006), através da comparação entre os resultados obtidos pelo MO e os obtidos pela Análise dos Campos (AC) de vento e vorticidade relativa. Por outro lado, uma avaliação da AC em identificar os VCANs foi feita, para o mesmo período, através da comparação entre os resultados desta e os obtidos usando Imagem de Satélite (IS) no canal espectral do vapor d'água. As AC apresentaram resultados satisfatórios e, portanto, o conjunto dos dados de reanálises do NCEP-NCAR foi aplicado ao MO. Foi observado um bom desempenho do $\mathrm{MO}$ em identificar os sistemas, bem como algumas características associadas, tais como: número de ocorrência, número de dias com atuação, posicionamento, direção de deslocamento e região de formação.

Palavras-chave: Vórtice ciclônico de altos níveis, método objetivo, validação, região Tropical Sul.
\end{abstract}

\begin{abstract}
OBJECTIVE METHOD IDENTIFICATION OF UPPER-TROPOSPHERIC CYCLONIC VORTICES IN THE SOUTH TROPICAL REGION: VALIDATION

In this study an Objective Method (OM) to identify Upper-Tropospheric Cyclonic Vortex (UTCV) in South Tropical region, based on relative vorticity and on horizontal wind flow of the wind around the system center was developed. The OM was elaborated using reanalysis data of the National Center for Environmental Prediction-National Centers for Atmospheric Research (NCEP-NCAR) and validated for a period of five years (from 2002 to 2006),by through a comparison between the OM results and the ones obtained through field analyses (FA) of wind and relative vorticity. On the other hand a FA for evaluation in identifying UTCV was made in the same period through by a comparison between these results and the ones obtained using Satellite Images on water vapor channel satellite pictures. The FA presented satisfactory results and, therefore set of NCEP-NCAR reanalysis was applied to the MO. A good OM performance was observed in identifying these systems, as well as some associated characteristics, such as, number of occurrence, number of days in action, positioning, displacement direction and region formation.
\end{abstract}

Keywords: upper-tropospheric cyclonic vortex, objective method, validation, South Tropical region.

\section{INTRODUÇÃO}

Existe uma grande necessidade em compreender a dinâmica, a termodinâmica e a climatologia sinótica dos sistemas transientes, pois um bom conhecimento do comportamento desses sistemas implicará em uma melhora na previsão de tempo de curto prazo, favorecendo subsídios de prevenção nos diferentes âmbitos de tomada de decisão.
Os Vórtices Ciclônicos de Altos Níveis (VCANs) são exemplos desses sistemas, que por sua persistência e deslocamento têm um papel importante no regime de precipitação de regiões dos trópicos e extratrópicos, além de contribuírem nas trocas de energia entre ambas as regiões (Ramirez, 1997). No Brasil, as regiões mais afetadas pelos VCANs são: Sul, Sudeste e Nordeste (Lourenço, 1996). Nesta última região, os sistemas se desenvolvem nas latitudes tropicais do Oceano Atlântico 
Sul (Kousky e Gan, 1981; Misha et al., 2001) e de acordo com experiências sinóticas podem ocasionar secas em algumas regiões ou inundações em outras, dependendo da posição sobre a área afetada (Silva, 2005) e das condições atmosféricas.

Os VCANs apresentam um movimento errático (Kousky e Gan, 1981; Simpson, 1951) e tempo de vida variado (Frank, 1970), o que torna a previsão de tempo complexa. Por isso, é de interesse da comunidade cientifica, a elaboração de um método totalmente objetivo que possa identificar e posicionar os vórtices da forma mais precisa possível. No entanto, a maior parte dos métodos objetivos elaborados são aplicáveis em áreas dos extratrópicos, como os estudos de Sinclair (1994), Fuenzalida et al. (2005) e Campetella e Possia (2006). Também existem outros métodos sofisticados que foram desenvolvidos para determinação de trajetórias percorridas por ciclones e anticiclones no Hemisfério Sul (HS), como nos estudos de Hodges (1994) e Hoskins e Hodges (2005).

Silva (2005) elaborou um método para identificação de VCANs no Nordeste do Brasil (NEB), mas não de forma totalmente objetiva, já que a análise da circulação fechada associada aos sistemas foi verificada com o uso de campos de linhas de corrente em $200 \mathrm{hPa}$. Neste estudo, um Método objetivo (MO) para identificar VCANs que atua na região Tropical Sul é apresentado, bem como sua validação em termos de posicionamento, número de ocorrência, número de dias com atuação, direção de deslocamento e região de formação. Com isso, se espera representar uma boa opção de aplicação em centros operacionais de tempo, como o Centro de Previsão de Tempo e Estudos Climáticos (CPTEC) do Instituto Nacional de Pesquisas Espaciais (INPE).

\section{DADOS E METODOLOGIA}

\subsection{Dados}

No presente estudo foram usados os seguintes conjuntos de dados:

(a) Reanálises do National Centers for Environmental Prediction - National Center for Atmosphere Research (NCEPNCAR) (Kalnay et. al., 1996) com resolução espacial de 2,5 de latitude por $2,5^{\circ}$ de longitude, freqüência temporal de 6 horas, nível padrão de $200 \mathrm{hPa}$ e período de 2002 a 2006 . As variáveis meteorológicas utilizadas são as componentes zonal (u) e meridional (v) do vento, e a área de estudo está limitada entre $72,5^{\circ} \mathrm{W}-7,5^{\circ} \mathrm{E}$ e $35^{\circ} \mathrm{S}-10^{\circ} \mathrm{N}$.

(b) Imagens dos satélites (IS) meteorológicos geoestacionários GOES-08 (2002 até maio de 2003) e GOES-12 (de junho de 2003 a 2006) no canal espectral do vapor d'água, disponíveis em intervalos de $3 \mathrm{em} 3$ horas e área limitada entre $140^{\circ} \mathrm{W}-20^{\circ} \mathrm{W}$ e $60^{\circ} \mathrm{S}-60^{\circ} \mathrm{N}$. Estas foram adquiridas da Divisão de Satélites Ambientais (DSA) do INPE. (c) Imagens dos mesmos satélites meteorológicos, canal espectral e período do item anterior. No entanto, a área compreende a região $140^{\circ} \mathrm{W}-0^{\circ}$ e $60^{\circ} \mathrm{S}-20^{\circ} \mathrm{N}$, e estas foram adquiridas no portal da National Oceanic and Atmospheric Administration (NOAA -http://www.nsof.class.noaa.gov/saa/ products/welcome) para o horário das 1200 UTC. Alguns dias não apresentaram imagens neste horário e então foram substituídas pelas mesmas imagens no horário das 1445 UTC. Os dados foram então submetidos à leitura e visualização no software Data Visualization Solutions (IDL), de forma que foram gradeadas em graus de latitude e longitude.

\subsection{Metodologia}

\subsubsection{Descrição dos Métodos Subjetivos}

\subsubsection{Imagens de Satélite}

O uso de imagens de satélite como método subjetivo para identificar VCANs que atuam no NEB, foi utilizado em vários estudos, tais como Kousky e Gan (1981), Gan (1983), Gan e Kousky (1986) e Ramirez (1997). Estes autores usaram estas imagens no canal espectral infravermelho e somente puderam identificar aqueles sistemas que apresentaram nebulosidade associada (denominado VCANs úmidos). No entanto, a experiência sinótica mostra a existência de alguns casos observados em imagens de satélite no canal do vapor d'água, que não estão associados ànebulosidade (denominados VCANs secos).

Bengtsson et al. (1982) fez o primeiro estudo para identificar características associadas aos VCANs através de imagens de satélite no canal do vapor d'água. Eles estudaram um caso de VCAN intenso, que se formou na costa leste do NEB em 22 de fevereiro de 1979, e se dissipou sobre o Brasil central no dia 28 de fevereiro. O VCAN apresentou características clássicas, tais como: centro seco e formação de nebulosidade na periferia.

Neste estudo, uma das identificações subjetivas dos VCANs foi feita utilizando as imagens dos satélites GOES-08 e GOES-12 no canal espectral do vapor d'água. A vantagem do uso destas imagens neste canal é que as regiões mais secas (tonalidade escura) ou mais úmidas (tonalidade clara), ao redor do centro do sistema, podem ser vistas perfeitamente, favorecendo assim ao acompanhamento do desenvolvimento do sistema desde seu estágio inicial, além da identificação de sistemas que não estão associados à nebulosidade (VCANs secos).

O procedimento aqui utilizado para identificar os vórtices, foi obtido através da localização nas imagens de satélite horárias de uma pequena região seca envolvida por uma região ciclônica de ar mais úmido, que poderia ou não estar associada à nebulosidade. Tais características deveriam aparecer ao longo da animação de pelo menos nove imagens consecutivas (dois dias de atuação), e sempre com característica de circulação 
ciclônica. A posição geográfica do VCAN foi obtida nas imagens de satélite diárias (1200 ou 1445 UTC), que estão gradeadas em graus de longitude e latitude.

\subsubsection{Análises dos Campos de Vento e de Vorticidade Relativa}

A segunda forma de identificação subjetiva do VCAN foi feita com campos de vento e vorticidade relativa no nível de 200 hPa para o período de 2002 a 2006, a partir de dados de reanálises do NCEP-NCAR. Neste caso, com os campos sobrepostos, os vórtices foram identificados através da localização de uma região que apresentasse circulação ciclônica ao longo de pelo menos dois dias (1200 UTC) consecutivos. As posições dos sistemas foram obtidas através das coordenadas geográficas do centro da circulação do vórtice em cada dia de atuação. Foi observado que a maioria dos vórtices com intensidade superior ao limiar proposto por Silva (2005) não conservavam a configuração fechada ao longo de dois dias. Desta forma, este limiar foi aplicado ao MO que está apresentado na seção seguinte.

\subsubsection{Descrição do MO}

O MO desenvolvido para identificar VCANs que atuam na região Tropical Sul foi baseado no método elaborado por Silva (2005), já que neste foi utilizado o nível de $200 \mathrm{hPa}$ e a vorticidade relativa em vez da altura geopotencial. Como normalmente é utilizado em outros métodos objetivos, o uso dessa última variável não é adequado para identificar distúrbios transientes na região tropical, porque a magnitude da amplitude do sistema tem a mesma ordem de grandeza dos erros cometidos na determinação do campo dessa variável (Palmén e Newton, 1969). A separação entre um VCAN e um Cavado em Altos Níveis (CAN) foi realizada de forma totalmente objetiva, sem a necessidade de analisar campos de linhas de corrente. Para isso, diversas etapas foram implementadas no algoritmo proposto por Silva (2005).

A idéia aplicada para separar os VCANs dos CANs pode ser entendida analisando o escoamento horizontal do vento nas configurações de um VCAN e de um CAN em torno do centro de máxima circulação ciclônica. As variações do sentido das componentes horizontais do vento em torno desse centro são semelhantes nos dois sistemas, até um ponto em que apenas a configuração do VCAN mostra um fechamento na circulação e a componente zonal do vento torna-se de leste no setor sul. Essa mudança não é observada na configuração de um CAN, pois não ocorre o fechamento da circulação e a componente zonal do vento permanece de oeste.

Baseado nestas configurações foi elaborado, em linguagem Fortran, o código para o MO, o qual obtém posição e intensidade dos VCANs que atuam na região Tropical Sul. Os passos para determinação dos parâmetros dos sistemas estão divididos em quatro etapas básicas: a primeira corresponde ao cálculo da vorticidade relativa em $200 \mathrm{hPa}$, a segunda obtém os mínimos desta variável, a próxima analisa a circulação em torno destes mínimos e a quarta etapa faz a seleção do VCAN. Esta última etapa é processada pelo MO, quando existem dois mínimos de vorticidade relativa envolvidos por uma mesma circulação ciclônica. Adiante as etapas estão descritas detalhadamente.

\section{(i) Etapa I - Cálculo da Vorticidade Relativa}

Utilizando dados das componentes horizontais do vento em $200 \mathrm{hPa}$, calcula-se a vorticidade relativa através do método das diferenças finitas centradas (Equação 1) em cada ponto do domínio compreendido entre $72,5^{\circ} \mathrm{W}-7,5^{\circ}$ E e $35^{\circ} \mathrm{S}-10^{\circ} \mathrm{N}$.

$\zeta(i, j, m)=\left(\frac{v(i+1, j, m)-v(i-1, j, m)}{2 d x}-\frac{u(i, j+1, m)-u(i, j-1, m)}{2 d y}\right)$

onde $\mathrm{i}$ e $\mathrm{j}$ são as posições do ponto de grade na direção $\mathrm{x}$ (longitude) e y (latitude) respectivamente, m é o passo de tempo, u é a componente zonal do vento e $\mathrm{v}$ a meridional, $\mathrm{dx}$ é distância entre dois pontos de grade na direção x e dy na y. Em cada passo de tempo $(\mathrm{m})$, o campo é pesquisado para encontrar os mínimos de vorticidade relativa, como mostrado na etapa II.

\section{(ii) Etapa II - Seleção dos Mínimos de Vorticidade Relativa}

A grade usada na determinação dos mínimos compreende o domínio entre $62,5^{\circ} \mathrm{W}-2,5^{\circ} \mathrm{W}$ e $25^{\circ} \mathrm{S}-0^{\circ}$, entretanto quatro pontos de grade das bordas ao limite dessa área são acrescidos para verificar as condições do escoamento horizontal em torno desses mínimos. São considerados como pontos de grade central, aqueles cujos valores de vorticidade relativa são inferiores ou iguais a $-2,5 \times 10^{-5} \mathrm{~s}^{-1}$ (Silva, 2005). Esse ponto é comparado aos valores desta variável dos oito pontos de grade vizinhos para verificar se realmente é o menor (mais negativo) do local. Caso seja o mínimo do local, o mesmo é passado para a etapa posterior, caso contrário, a análise é passada para o ponto de grade seguinte.

\section{(iii) Etapa III - Avaliação da Circulação do Vento Horizontal em Torno do Mínimo}

Nesta etapa do MO, o mínimo é submetido à avaliação da circulação horizontal do vento ao redor do sistema. Nessa análise, o mínimo de vorticidade relativa encontrado representa a intensidade do sistema, que geralmente encontra-se um pouco 
defasada do centro da circulação do vento ao redor do centro do VCAN. Desta forma, o escoamento horizontal do vento em torno desse mínimo, é avaliado nos quatro quadrantes, considerando os vinte e quatro pontos de grade mais próximos do mínimo, ou seja, esta distância do mínimo correspondente a dois pontos de grade. Cada quadrante apresenta uma condição para a direção das componentes horizontais do vento, como pode ser visto na Tabela 1. Em determinados casos, a matriz de análise dessas condições pode ser expandida em até mais dois pontos de grade, totalizando uma distância do mínimo correspondente a quatro pontos de grade, se necessário à satisfação do quadrante. $\mathrm{O}$ uso da expansão na matriz de análise foi necessário para identificação dos VCANs alongados, encontrados ao longo da identificação.

Ao ser satisfeita a condição em cada quadrante, é feita uma captura da posição dos pontos que constituem a matriz de análise e da contabilidade dos mesmos. Quando o mínimo finaliza a passagem por todos os quadrantes, é considerado como válido para a seleção dos VCANs (Etapa IV), e os seguintes parâmetros são armazenados: intensidade, posição geográfica em graus, soma dos números de pontos de análise de todos os quadrantes (NP) e número de mínimos válidos (NV).

\section{(iv) Etapa IV - Seleção do VCAN}

A idéia desta etapa é baseada nos casos de mais de um mínimo de vorticidade ciclônica válido envolvidos por uma mesma circulação, ou seja, o sistema apresenta mais de um núcleo de vorticidade mínima. Desta forma, o pré-requisito utilizado pelo método para definir como o VCAN é selecionar um desses núcleos através de comparações entre a posição de todos os pontos de grade usados na análise matricial de cada núcleo. Esse processo é feito através de uma varredura dos núcleos encontrados (mínimos válidos), definidas como núcleo que está sendo analisado e a dos núcleos que estão sendo comparados, com suas respectivas posições dos pontos de grade associadas. Quando pelo menos um ponto de grade do núcleo analisado coincide com um ponto do núcleo comparado, significa que os núcleos estão envolvidos por uma mesma circulação. Dessa forma, o núcleo mais intenso (vorticidade mais negativa) é selecionado. Nos casos em que os núcleos não estão envolvidos por uma mesma circulação,

Tabela 1 - Condições do vento horizontal em cada quadrante, necessárias à configuração de um VCAN.

\begin{tabular}{|l|l|}
\hline Quadrante I & $\mathrm{u}>0$ e $\mathrm{v}<0$ \\
\hline Quadrante II & $\mathrm{u}>0$ e $\mathrm{v}>0$ \\
\hline Quadrante III & $\mathrm{u}<0$ e $\mathrm{v}>0$ \\
\hline Quadrante IV & $\mathrm{u}<0$ e $\mathrm{v}<0$ \\
\hline
\end{tabular}

são denominados como "VCANs isolados", visto que cada núcleo apresenta circulação independente. Após a passagem por todas as etapas do método, as posições e intensidades dos vórtices são armazenadas.

\subsubsection{Avaliação das Análises}

Já que o MO foi desenvolvido com dados do NCEPNCAR, um importante passo, antes de validar o mesmo para o período de cinco anos (2002 a 2006), foi avaliar se esse conjunto de dados representa satisfatoriamente as principais características (posição, número de ocorrência, período de vida e região de formação) dos sistemas. A avaliação dos dados foi feita através da comparação entre as posições dos sistemas obtidas pela $\mathrm{AC}$ e as obtidas pela IS, desta forma, garantindo uma boa aplicação do MO com o conjunto de dados do NCEPNCAR. Durante a validação do MO, o número de ocorrência, duração e as posições dos sistemas obtidas pela $\mathrm{AC}$ foram comparadas às obtidas pelo MO. O cálculo das defasagens entre as posições dos VCANs foi feito através da diferença entre a latitude (longitude) da análise de identificação avaliada e a latitude (longitude) da que foi considerada como verdade. Com a comparação entre as três análises de identificação, obtiveram-se o número de VCANs e o número de dias com atuação destes sistemas, considerando-se apenas os vórtices formados a oeste de $20^{\circ} \mathrm{W}$ do domínio em estudo. Esta região foi escolhida porque o uso das imagens horárias dos satélites GOES-8 e GOES-12, adquiridas da DSA/INPE, apresentam domínio aproximadamente nesta região.

\section{RESULTADOS E DISCUSSÃO}

\subsection{Comparação entre Imagens de Satélite no Canal do Vapor D’água e os Campos de Vento e de Vorticidade Relativa}

O período de avaliação é de 2002 a 2006, porém aqui é mostrada parte da avaliação através das comparações da distribuição espacial dos VCANs, obtidas pelas análises subjetivas para o verão (NDJF) de 2002-2003, por ser o que apresentou maior número (18) de VCANs do período. Vale lembrar que em todas as comparações feitas com a IS, são contabilizados os VCANs formados a oeste de $20^{\circ} \mathrm{W}$, devido a área de cobertura da IS, isto significa que pode ser encontrado um número inferior de vórtices se comparado aos resultados obtidos durante a avaliação dos dados do NCEP (comparação apenas entre $\mathrm{AC}$ e $\mathrm{MO}$ ), já que neste caso, a área é maior. Como avaliação geral, o número de VCANs e o número de dias com atuação destes sistemas são mostrados para todo o período avaliado na Tabela 2. 
A Figura 1 mostra a distribuição espacial dos VCANs identificados através da AC e da IS para o verão 2002/2003. Observam-se semelhanças entre a distribuição dos VCANs obtidas pelas duas formas de análises ao longo da região que abrange a costa do NEB e oceano Atlântico Sul adjacente. No entanto, uma defasagem é observada entre as posições, visto que ambas as análises utilizam critérios e dados distintos para localização dos VCANs. Considerando o período analisado
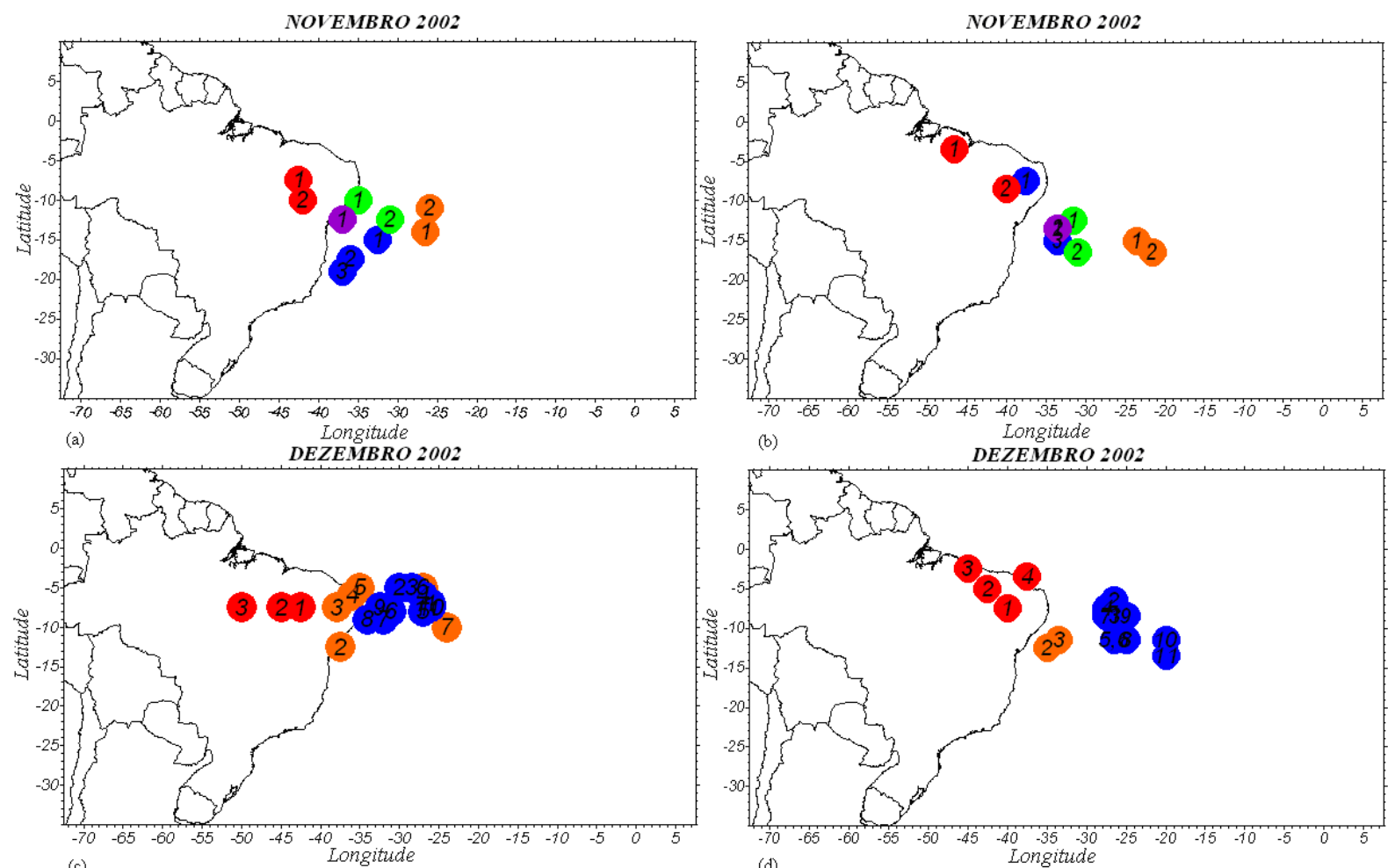

(c)

JANEIRO 2003
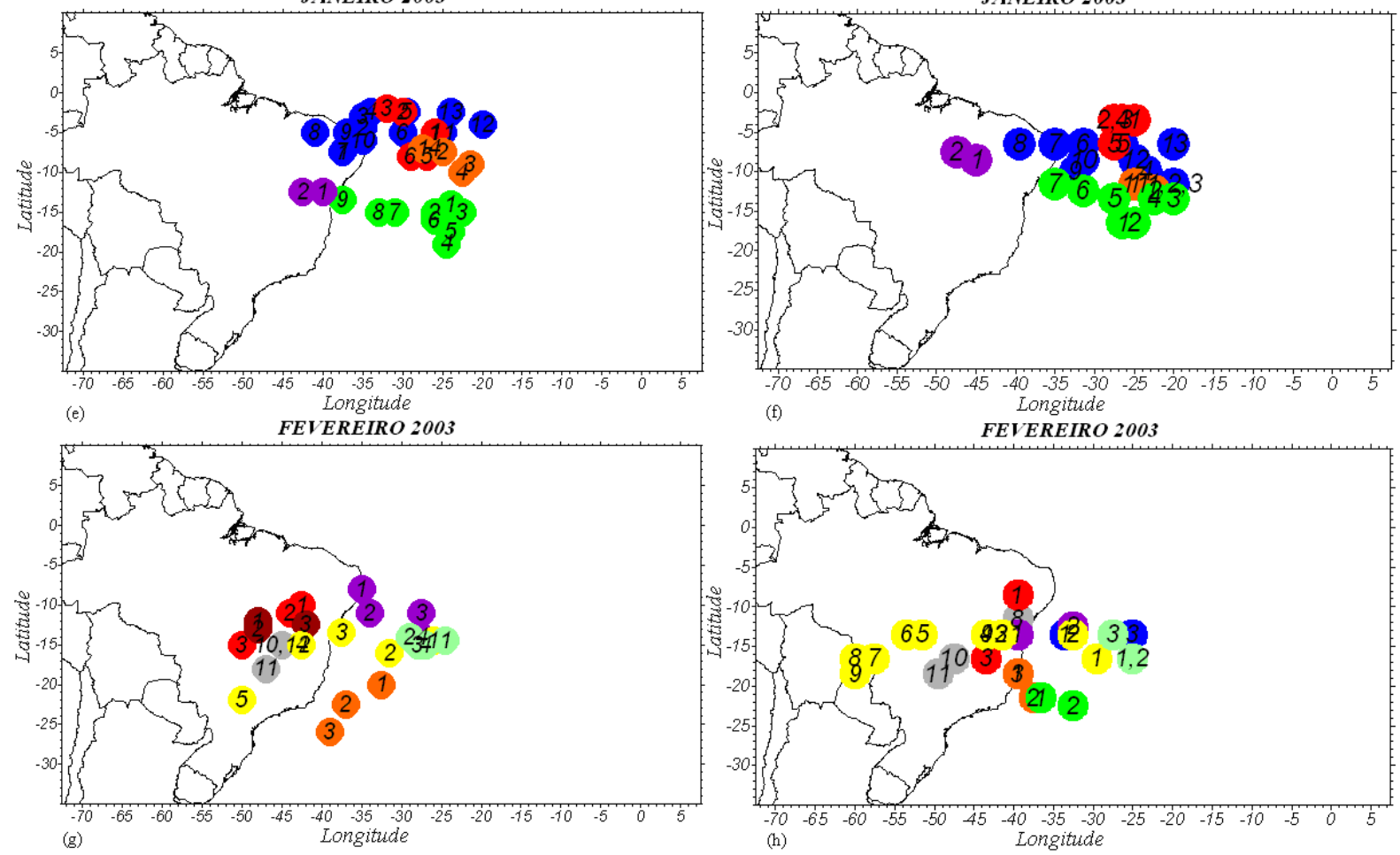

Figura 1 - Distribuição espacial dos VCANs. À esquerda: obtida pela IS; à direita: obtida pela AC, para o verão de 2002-2003. A cor indica o VCAN e a ordem numérica informa a seqüência de atuação do mesmo. 
Tabela 2 - Número de VCANs obtidos pelos três métodos de análises: IS, MO e AC. Os números entre parênteses indicam número de dias com VCANs.

\begin{tabular}{|c|c|c|c|c|c|c|c|c|c|c|c|c|c|c|c|c|c|c|}
\hline MÊS & \multicolumn{3}{|c|}{2002} & \multicolumn{3}{|c|}{2003} & \multicolumn{3}{|c|}{2004} & \multicolumn{3}{|c|}{2005} & \multicolumn{3}{|c|}{2006} & \multicolumn{3}{|c|}{ TOTAL } \\
\hline & IS & MO & $\overline{\mathrm{AC}}$ & IS & MO & $\mathrm{AC}$ & IS & MO & $\mathrm{AC}$ & IS & MO & $\mathrm{AC}$ & IS & MO & $\mathrm{AC}$ & IS & MO & $\mathrm{AC}$ \\
\hline FEV & $5(27)$ & $7(28)$ & $7(29)$ & $6(26)$ & $7(29)$ & $7(29)$ & $4(24)$ & $4(20)$ & $4(20)$ & $2(8)$ & $2(13)$ & $2(15)$ & $2(18)$ & $3(19)$ & $3(19)$ & $19(103)$ & $23(109)$ & $23(112)$ \\
\hline $\mathrm{ABR}$ & $2(12)$ & $2(12)$ & $2(12)$ & $4(12)$ & $4(12)$ & $4(12)$ & $4(8)$ & $5(12)$ & $5(12)$ & $2(6)$ & $2(6)$ & $2(6)$ & $1(5)$ & $1(2)$ & 1(2) & $13(43)$ & $14(44)$ & $14(44)$ \\
\hline MAI & & & & & & & & & & $2(5)$ & 1(2) & $1(2)$ & & & & $2(5)$ & 1(2) & $1(2)$ \\
\hline JUN & 1(3) & 1(3) & 1(3) & & & & & & & & & & & & & $1(3)$ & 1(3) & $1(3)$ \\
\hline SET & $2(5)$ & $2(5)$ & $2(5)$ & & & & $3(8)$ & $3(7)$ & $3(7)$ & & & & & & & $5(13)$ & $5(12)$ & $5(12)$ \\
\hline OUT & $1(6)$ & $1(7)$ & 1(7) & $2(8)$ & $2(5)$ & $2(5)$ & $3(8)$ & $4(13)$ & $4(13)$ & $3(13)$ & $5(17)$ & $5(18)$ & & 1(3) & 1(3) & $9(35)$ & $13(45)$ & $13(46)$ \\
\hline Nov & $5(10)$ & $5(10)$ & $5(10)$ & $2(8)$ & 2(3) & 2(3) & $3(8)$ & $3(7)$ & $3(7)$ & $3(16)$ & $2(9)$ & $2(9)$ & $5(16)$ & $5(22)$ & $5(22)$ & $18(58)$ & $17(51)$ & $17(51)$ \\
\hline DEZ & $2(21)$ & $2(17)$ & $2(17)$ & $2(20)$ & $2(16)$ & $2(16)$ & $3(18)$ & $4(20)$ & $4(21)$ & $3(20)$ & $3(18)$ & $3(19)$ & $5(17)$ & $7(25)$ & $7(25)$ & $15(96)$ & $18(96)$ & $18(98)$ \\
\hline ANO & $24(115)$ & $26(119)$ & $26(120)$ & $26(124)$ & $28(116)$ & $28(115)$ & $25(104)$ & $28(106)$ & $28(107)$ & $21(100)$ & $26(113)$ & $25(117)$ & $22(96)$ & $28(108)$ & $28(108)$ & 118(539) & $136(562)$ & \begin{tabular}{|l}
$135(567)$ \\
\end{tabular} \\
\hline
\end{tabular}

e tomando-se como exemplo a Figura 1, observa-se que na maioria dos meses, a AC contabiliza o mesmo número de VCANs do que a IS, com exceção de alguns casos, como o em verde escuro e em azul da Figura 1h, na qual a AC identifica a mais do que a IS, e o caso em marrom (Figura $1 \mathrm{~g}$ ) que não é identificado pela AC. Outra diferença pode ser vista na Figura 1d, neste caso a AC subestima o período de vida dos VCANs com a cor em laranja, que foi formado em novembro de 2002, próximo à costa do NEB (Figura $1 \mathrm{~b}$ - cor em roxo). Este VCAN deveria apresentar seis dias (Figura 1c) de atuação em dezembro, no entanto a AC só identificou dois dias (Figura 1d). Este é um dos casos identificados no período (2002-2006) que a AC mostra problema em estimar o período de vida dos VCANs, além de outros exemplos aqui apresentados como os casos nas cores em azul e vermelho da Figura 1d, os casos em vermelho, laranja e verde da Figura if e os casos em cinza, amarelo, verde claro e roxo da Figura $1 \mathrm{~h}$.

A Tabela 2 mostra o número anual e mensal de VCANs obtidos pelo $\mathrm{MO}$ e os métodos subjetivos (IS e AC) para todo o período avaliado, bem como o número de dias com VCANs. Através da avaliação anual do número de VCANs obtido pela $\mathrm{AC}$, nota-se que esta superestima um número total em 17 casos, já que pela IS foram identificados 118 VCANs. AAC obteve um número mais satisfatório no ano de 2002 e 2003, apresentando uma diferença de 2 casos com relação a IS, já a maior diferença ( 6 casos) pode ser vista no ano de 2006. O número anual de dias com vórtice obtido pela $\mathrm{AC}$, segundo esta tabela, totaliza 567 dias, correspondendo a uma diferença de 28 dias, quando comparado aos 539 dias obtido pela IS. Fazendo uma avaliação anual, observa-se que em 2004, a AC representou mais satisfatoriamente este número, mostrando uma diferença de 3 dias, já em 2005, esta diferença foi a maior e corresponde a 17 dias. A diferença nestes dois últimos anos foi superestimada, assim como nos demais anos, exceto em 2003 em que a AC identificou um número inferior correspondente a 9 dias com VCANs.

A avaliação da Tabela 2, por estação do ano, mostra que a AC identificou um número superior de VCANs em todas as estações, principalmente no verão, com uma diferença de 10 casos, já no inverno, a AC identificou um caso a mais (em JUL de 2005). As diferenças de casos observados entre os dois métodos na primavera e no outono correspondem a 3 casos. Com relação ao número de dias com VCANs, a AC também mostrou um número superior em todas as estações. $\mathrm{O}$ verão e o outono apresentaram maiores diferenças, correspondendo a 13 e a 9 dias, respectivamente. Já o inverno e a primavera mostraram uma diferença correspondente a 3 dias.

Devido às diferenças encontradas entre as análises subjetivas, buscou-se exemplificar o motivo destas através da Figura 2, a qual apresenta dias com atuação de VCANs em situações distintas. A primeira situação (Figuras 2a, 2c) se refere ao não fechamento da circulação do VCAN na AC em alguns dias, já a segunda situação (Figuras $2 b, 2 d$ ) apresenta um caso em que a AC mostra o VCAN que não existe na IS. Analisando a Figura 2c, nota-se que a IS identifica claramente o VCAN, bem como o centro em torno de $10^{\circ} \mathrm{S}, 27,5^{\circ} \mathrm{W}$, porém a AC (Figura 2a) mostra uma região de cavamento coincidente à região de atuação do VCAN. A partir deste dia (16 de fevereiro), a AC não apresenta fechamento da circulação associado ao VCAN e por isso o período de vida do mesmo é menor do que o da IS.

As Figuras $2 \mathrm{~b}$ e $2 \mathrm{~d}$ apresentam a segunda situação exposta acima. A AC identifica um VCAN com centro em $0^{\circ}$ e $32.5^{\circ} \mathrm{W}$ (Figura 2b) que não é visto na IS (Figura 2d). Nesta região, a IS mostra nebulosidade associada a um cavado em altos níveis que se encontra sobre o Oceano Atlântico. Os dados de reanálises do NCEP-NCAR em regiões oceânicas do equador e proximidades, em algumas situações, mostram circulações 

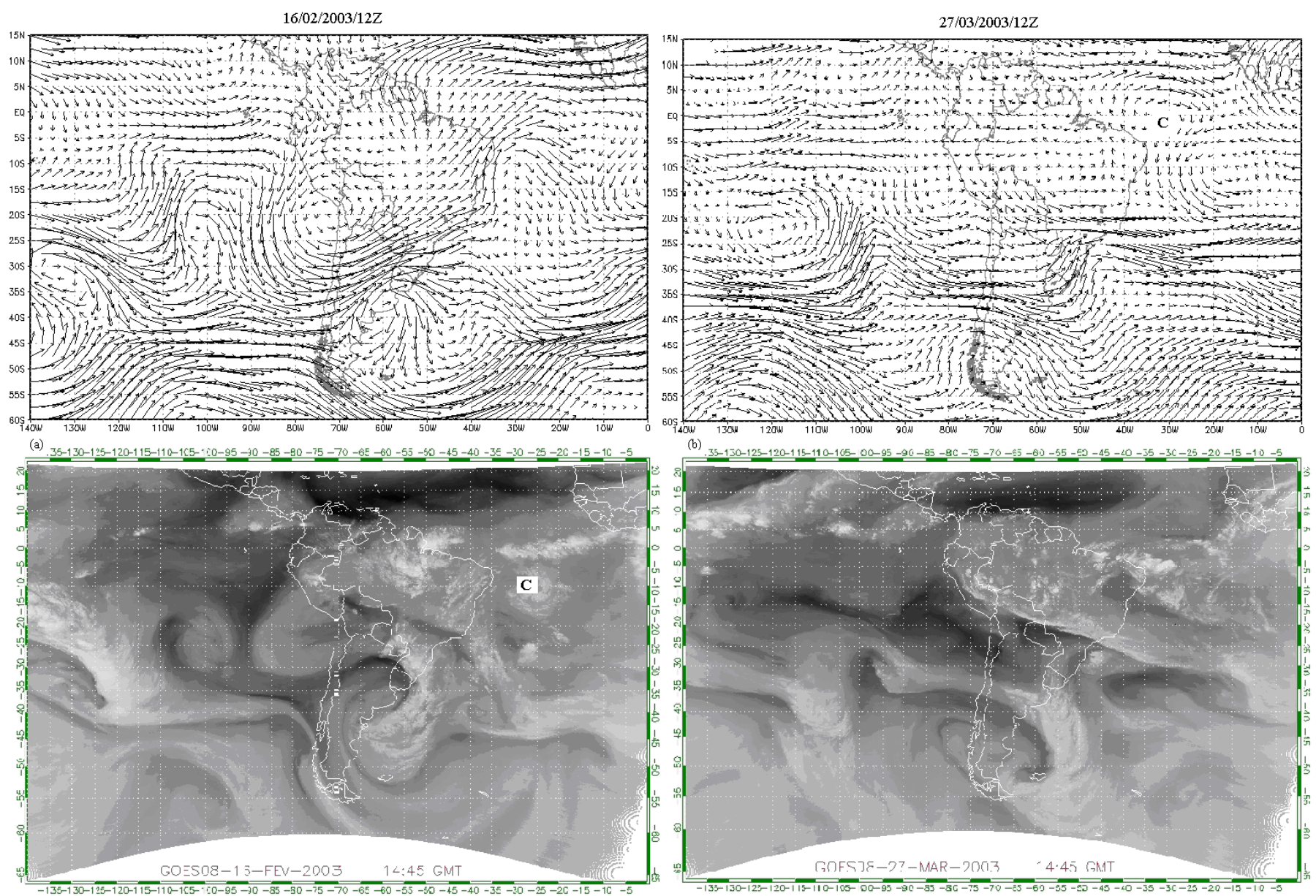

(c)

(d)

Figura 2 - Campos de vento em 200 hPa às 12:00 UTC e Imagens do satélite GOES-08 às 14:45 UTC nos dias (a) e (c) 16/02/03 e (b) e (d) 27/03/03. "C" indica o centro do VCAN.

ciclônicas associadas a vórtices fracos que chegam ao Hemisfério Norte. Provavelmente, poderia ser um problema de estimativa do vento em $200 \mathrm{hPa}$ através de informações de satélite, já que normalmente há falta de dados de radiossondagens sobre o Oceano Atlântico. Assim, a identificação dos VCANs com dados de reanálises do NCEP-NCAR nestas regiões, pode ocasionar defasagens entre a AC e a IS quanto ao número de VCANs e/ou ao período de vida dos mesmos que se formam ou se deslocam nestas regiões.

A Figura 3 mostra a defasagem entre as posições dos VCANs obtida pela AC com relação a IS para o ano de 2003. Nesta figura são consideradas as posições nos dias em que os dois métodos identificaram o VCAN, já que o objetivo é avaliar o quanto a AC posiciona corretamente esse sistema. Nota-se que o maior número de defasagens na posição dos VCANs apresenta variação longitudinal de -5 a 10 graus e latitudinal de -5 a 5 graus, indicando posições aproximadas entre as duas análises subjetivas e, portanto boa identificação destas pela AC. A maior defasagem encontrada no gráfico (Figura 3) é longitudinal com valor de $-21,5^{\circ}$, enquanto que a maior defasagem latitudinal apresenta valor de $14,6^{\circ}$, isto significa que a $\mathrm{AC}$ identificou esses VCANs que não foram observados na IS.

As regiões do primeiro (I) e quarto (IV) quadrante deste gráfico apresentam um número maior de posições defasadas do que nos demais quadrantes (II e III). Isto significa que a AC mostrou tendência em posicionar os VCANs mais para leste, já que grande parte dos VCANs mostrou defasagem latitudinal positiva. Longitudinalmente, a AC mostra aproximadamente o mesmo número de posições dos VCANs defasadas tanto para sul, quanto para norte da posição que deveria apresentar usando a IS. O menor número de defasagens pode ser visto no quadrante III, indicando que neste ano, a AC mostra pouca tendência em posicionar os VCANs simultaneamente mais para leste e sul de sua posição real (IS). Algumas defasagens são vistas no extremo esquerdo dos quadrantes II e III e no extremo direito do quadrante IV, mostrando posições mais distantes entre a AC e a IS. 


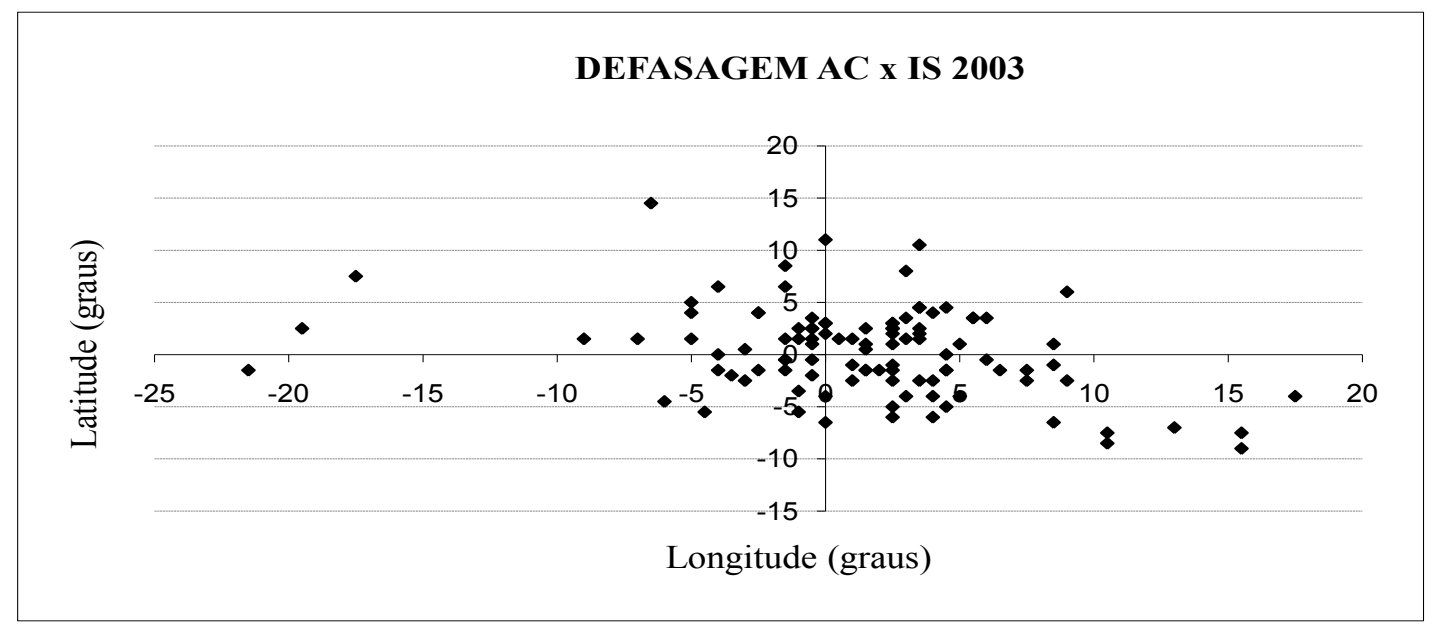

Figura 3 - Defasagem (graus) das posições dos VCANs obtidas pela AC com relação a IS em 2003.

\subsection{Validação do MO}

Para avaliação do $\mathrm{MO}$, o período é o mesmo usado nas comparações descritas anteriormente (2002 a 2006). Os resultados apresentados, quanto à distribuição espacial dos sistemas, são comparados com os resultados obtidos pela $\mathrm{AC}$ para os quadrimestres NDJF (2002-2003) e NDJF (2003-2004), por serem os verões com maior (27 casos) e menor (15 casos) número de VCANs, respectivamente. Essas comparações têm um importante sentido que é testar o funcionamento do MO com o conjunto de dados do NCEP-NCAR, sendo a AC, a forma realística de identificação.

As Figuras 4 e 5 apresentam os resultados obtidos para os dois verões de 2002-2003 e 2003-2004, respectivamente. Embora o MO tenha apresentado um bom desempenho na identificação dos sistemas, uma defasagem é vista entre as posições encontradas pelos métodos (AC e $\mathrm{MO}$ ), isso porque o MO posiciona o sistema através do mínimo de vorticidade relativa, já a AC posiciona pelo centro da circulação do sistema, e nem sempre este centro coincide com o ponto de mínima vorticidade relativa. Como conseqüência, diferenças podem ser notadas entre as características do sistema obtidas por estes métodos de identificação. Isto pode ser visto analisando os casos com as cores em vermelho e em azul das Figuras 4a e 4b. O MO identifica o deslocamento do VCAN com a cor em azul com direção para sul (Figura 4a), sendo que esse deveria mostrar deslocamento na direção para sudeste (Figura 4b). Já no caso com a cor em vermelho (Figura 4a), o MO identifica corretamente a direção do deslocamento para sudeste, assim como na Figura 4b. Nota-se que para estes dois casos de VCANs, o MO (Figura 4a) tende a subestimar a distância percorrida pelos mesmos, além das posições de origem e dissipação dos sistemas apresentarem ligeiras diferenças em graus com relação a AC (Figura 4b).

Em alguns poucos casos, os quais possuem período de vida curto (dois dias), a AC só identificou um dia de atuação do sistema, já que a circulação no outro dia não estava fechada e como são consideradas as posições dos vórtices que perduram dois ou mais dias, estes casos foram desconsiderados durante a contagem na AC. Como exemplo, destaca-se o caso com a cor em rosa da Figura 4e, no qual o MO identifica a mais.

Algumas características padrões dos sistemas estão bem representadas pelo $\mathrm{MO}$, como direção do deslocamento e região de formação. Segundo Gan (1983), a formação da maioria dos vórtices ocorre na área que abrange a costa do NEB e Oceano Atlântico Sul adjacente, compreendendo a faixa latitudinal de $0^{\circ}$ a $20^{\circ} \mathrm{S}$, exceto alguns VCANs, como o caso com a cor em verde escuro (Fig. 4g) cuja região de formação é ao sul de $20^{\circ} \mathrm{S}$, e o caso com cor em marrom (Fig. 4g) que apresenta origem em $0^{\circ}$. Nas áreas de maior ocorrência de formação há concentração desses sistemas, indicando que muitos casos têm um lento deslocamento.

Os meses de janeiro e fevereiro de 2003 apresentam maior número de ocorrência de vórtices (Figura 4), concordando com os estudos de Kousky e Gan (1981), Gan (1983) e Ramirez (1997), já na Figura 5, os meses de dezembro de 2003 e fevereiro de 2004 apresentam maior número de casos, nos quais é observado um número total de VCANs inferior ao da Figura 4, principalmente porque houve uma redução não freqüente do número de VCANs no mês de janeiro de 2004. Em ambas as Figuras, o MO mostra o mês de novembro como o que apresenta menor número de sistemas.

Através da avaliação do número anual de VCANs da Tabela 2, nota-se que o MO identifica apenas um caso a mais do 

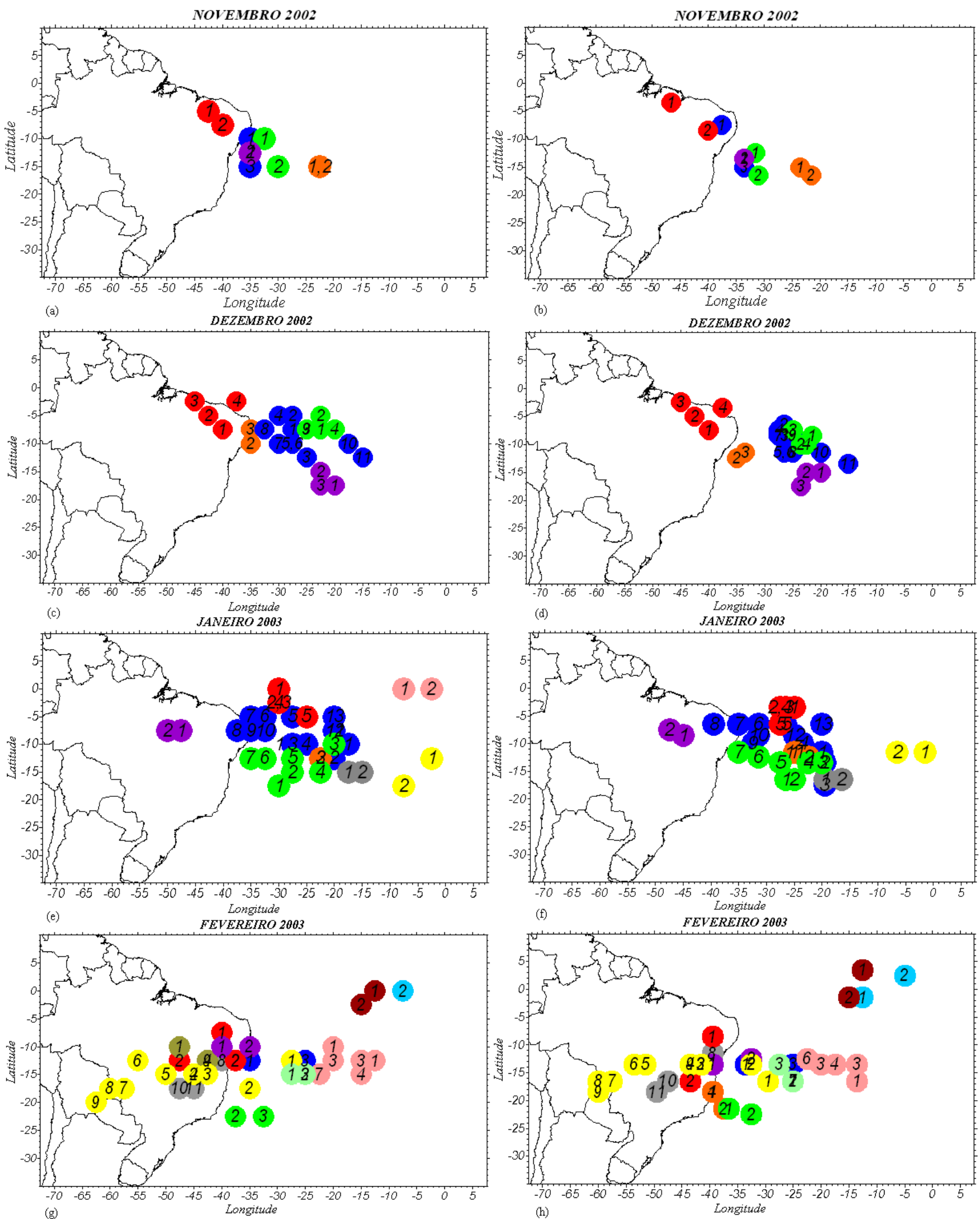

Figura 4 - Como a Figura 1, sendo à esquerda: obtida pelo MO, à direita: obtida pela AC. 

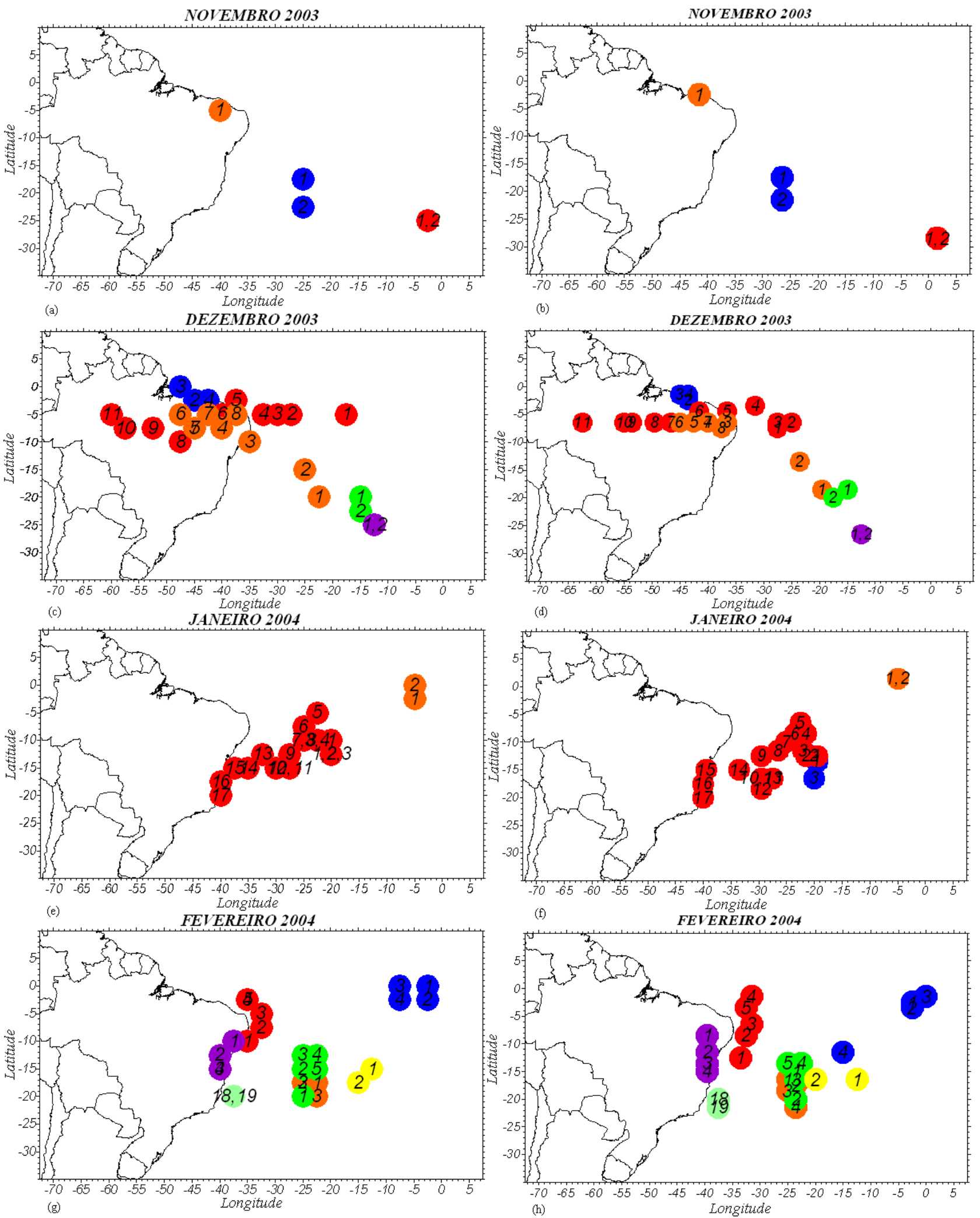

Figura 5 - Como Figura 4 para o verão de 2003-2004. 
que a AC no ano de 2005, ao longo do período avaliado (20022006). Quanto ao número anual de dias com vórtice durante o período, o MO subestima 5 dias. Destes dias, o ano de 2005 foi o que apresentou maior diferença (4 dias). Já no ano de 2006, o $\mathrm{MO}$ mostrou o mesmo número de dias com VCANs que a AC. A diferença para os anos de 2002, 2003 e 2004 corresponde a 1 dia. A avaliação do número de VCANs por estação do ano mostra que este número obtido pelo $\mathrm{MO}$ foi mais satisfatório no verão, já que apresentou o mesmo número (64 casos) de VCANs que a AC. A primavera foi a que apresentou maior diferença correspondente a 3 casos. As estações de outono e inverno mostraram diferença de 2 casos e 1 caso, respectivamente. Quanto à avaliação do número de dias com VCANs, nota-se que o MO superestima este número no inverno, já nas demais estações do ano, ocorre subestimativa deste número, sendo a maior diferença mostrada no verão com 5 casos.

A Figura 6 mostra a defasagem da posição dos VCANs identificada pelo MO com relação a AC para o ano de 2003, lembrando que esta última é considerada como verdade. A maioria das defasagens encontra-se no intervalo de valores longitudinais de $-2,5$ a 2,5 graus e com valores latitudinais de -4 a 4 , indicando que as posições dos sistemas são bem representadas pelo MO, já que a maioria das defasagens está próxima ao centro do gráfico. Nota-se que o número de defasagens encontra-se aproximadamente distribuído nos quadrantes (I, II, III e IV). Isto significa que não é visto clara direção de deslocamento da posição dos VCANs obtida pelo MO com relação a AC, como verificado nas comparações obtidas entre a AC e a IS (Figura 3). Porém, a Figura 6 mostra que os maiores valores de defasagens obtidos são longitudinais, assim como visto na Figura 3. Isto pode ser observado através de alguns pontos extremos nos quadrantes I e $\mathrm{IV}$, indicando posições obtidas pelo $\mathrm{MO}$, os quais tendem a defasar mais significativamente na direção longitudinal do que latitudinal.

\subsection{Comparação entre os Métodos de Análise de Identificação}

Considerando os resultados apresentados na Tabela 2 e utilizando a IS como verdade terrestre, nota-se que existe uma homogeneidade no número total de casos por ano, ao longo do período, entre as análises dos três métodos de identificação e um bom desempenho do MO, embora este tenha superestimado com diferenças correspondentes a dois casos em 2002 e em 2003, três em 2004, cinco em 2005 e seis em 2006. A AC apresenta número total anual similar ao $\mathrm{MO}$ e, portanto superestima os resultados obtidos pela IS em todos os anos e com mesma diferença, exceto em 2005 que corresponde a quatro casos. Em 2002 e 2003, os resultados do $\mathrm{MO}$ e da $\mathrm{AC}$ são mais satisfatórios, já que apresentam diferença de dois casos quando comparados a IS.

O número de VCANs, obtido pela IS em alguns meses, foi inferior aos identificados com as demais análises durante o período de estudo (2002-2006), sendo mais freqüente no primeiro quadrimestre JFMA, como exemplo em fevereiromarço de 2002, fevereiro-março de 2003, abril de 2004 e janeiro de 2005 e janeiro-fevereiro de 2006. Em alguns destes exemplos, as análises avaliadas identificaram VCANs no equador e proximidades que não existem na IS. Em outras situações, a IS não mostra escoamento com rotação horária (circulação ciclônica) durante as animações e assim estes casos não são considerados como VCANs, desta forma os métodos (MO e $\mathrm{AC)}$ identificaram um número superior.

Em outros meses, a AC e o MO subestimam o número de VCANs, como exemplos em janeiro de 2002, maio e novembro de 2005, que apresentam diferença de um caso por estas duas formas de análises. Nestes exemplos, o VCAN apresenta-se bem configurado na IS, porém na $\mathrm{AC}$ não é observada configuração fechada (Figura 2a) e no MO, alguma condição do critério adotado não é satisfeita, e portanto, o caso não é considerado como VCAN. Estas justificativas pelos quais o $\mathrm{MO}$ e a $\mathrm{AC}$ subestimam ou superestimam o número de VCANs, são as mesmas para explicar as diferenças encontradas no número de dias com VCANs, apresentado na Tabela 2. Este número de dias com VCANs obtido pelas análises (MO e AC) tende a apresentar diferenças mais significativas do que o número destes sistemas obtido por estas análises. Considerando a análise anual deste número, nota-se que apenas no ano de 2002, as duas análises avaliadas mostram um número de dias inferior destes, correspondentes a 4 dias pelo $\mathrm{MO}$ e 5 dias pela AC. Nos demais anos, são obtidos número de dias superiores, com diferenças de no máximo 13 dias em 2005 obtidas pelo MO, e 17 dias neste mesmo ano obtidas pela AC. A menor diferença encontrada foi em 2004 e corresponde a 2 dias obtida pelo MO e 3 dias obtida pela AC.

Fazendo uma avaliação, por estação do ano, do número de VCANs e do número de dias com atuação destes sistemas, nota-se que este primeiro número mostra que a $\mathrm{AC}$ e o $\mathrm{MO}$ superestimam em todas as estações do ano, exceto na primavera, em que o MO identifica o mesmo número de VCANs que a IS, correspondendo a 32 casos. No inverno, a diferença corresponde a 2 casos obtida pelo MO e 1 caso obtida pela AC. Já no verão, a diferença é de 10 casos pelas duas análises avaliadas. O número de dias com VCANs mostra um número superior obtido pelas duas análises, sendo a diferença maior no verão e no outono, correspondentes a 8 dias pelo $\mathrm{MO}$ e 13 dias pela AC durante o verão, e 8 dias pelo $\mathrm{MO}$ e 9 dias pela $\mathrm{AC}$ durante o outono. $\mathrm{O}$ inverno apresentou diferença de 5 e 3 dias obtida pelo $\mathrm{MO}$ e pela $\mathrm{AC}$, respectivamente.

Verificou-se que as diferenças no número de dias com atuação dos VCANs obtidas pelo $\mathrm{MO}$ e pela $\mathrm{AC}$, ocorreram com maior freqüência no período de dissipação dos sistemas avaliados. De 58 dos VCANs avaliados, 28 casos mostraram diferença no período de dissipação, 19 no período de formação e 
os 11 casos restantes, tanto no início, quanto no final do período de vida dos VCANs. Isto significa que as análises avaliadas tendem a manter os sistemas por mais dias, já que os dados de reanálises do NCEP-NCAR possuem um "first guess" de seis horas que pode apresentar falta de dados de vento observados na região do VCAN, e prolongar o período de atuação do sistema.

\section{CONCLUSÕES}

Foi desenvolvido um MO que identifica VCANs formados no nível de $200 \mathrm{hPa}$ na região Tropical Sul, a partir de um limiar $\left(-2,5.10^{-5} \mathrm{~s}^{-1}\right)$ de vorticidade relativa (Silva, 2005) e de condições pré-estabelecidas da circulação horizontal do vento ao redor do centro do sistema. Um processo de validação foi feito com o MO, a partir de dados de reanálises do NCEP-NCAR para o período de cinco anos (2002-2006). A identificação dos sistemas obtida pelo MO foi comparada com a obtida pela AC, considerada como verdade nesta fase, já que o objetivo foi a aplicação do MO utilizando este conjunto de dados. Por outro lado, a AC foi avaliada para o mesmo período através das comparações dos resultados da mesma com as obtidas pela IS, neste caso, esta última foi considerada como verdade.

As comparações mostraram boa destreza da AC em identificar os vórtices, porém as posições obtidas apresentaram pequenas defasagens, devido aos critérios adotados para identificação serem distintos. A avaliação anual do número de VCANs mostrou que a AC identificou 17 vórtices a mais do que a IS, considerando os cinco anos (2002-2006). Enquanto que o número de dias com VCANs apresentou diferença de 28 dias a mais neste período. A avaliação, por estação do ano, mostrou que a AC identificou um número superior de VCANs em todas as estações, principalmente no verão, com uma diferença de 10 casos. Com relação ao número de dias com VCANs, a AC também mostrou um número superior em todas as estações, sendo a maior diferença no verão correspondente a 13 dias.

As diferenças entre a $\mathrm{AC}$ e a IS quanto ao número e/ ou ao período de vida dos VCANs, encontrados em regiões oceânicas do equador e proximidades, podem estar associadas a problemas de estimativa de vento em $200 \mathrm{hPa}$ através de informações de satélite, já que geralmente nas regiões oceânicas há falta de dados de radiossondagens. Desta forma, pode ocorrer perda de informações nos dados de reanálises do NCEP-NCAR para estimar a circulação horizontal fechada do vento, associada aos vórtices.

Por sua vez, o MO mostrou ligeiras defasagens entre as posições dos vórtices obtidas na maioria dos casos, porque o mesmo posicionou o sistema através do mínimo de vorticidade relativa e a AC pelo centro da circulação do sistema e nem sempre este centro coincidiu com o ponto de mínima vorticidade relativa, pois a maioria dos VCANs não tem uma estrutura horizontal simétrica. A avaliação anual do número de VCANs mostrou que o MO identificou apenas 1 caso a mais durante o período, já na análise do número anual de dias com vórtice, o MO subestimou 5 dias. A avaliação do número de VCANs por estação do ano mostra que este número obtido pelo MO foi satisfatório em todas as estações do ano, já que apresentaram o mesmo número de VCANs, exceto no inverno onde o MO identificou 1 caso a mais. Quanto à avaliação do número de dias com VCANs, o MO superestimou este número no inverno, já nas demais estações do ano, ocorreu subestimativa deste número, sendo a maior diferença de 5 dias mostrada no verão.

Considerando todo o período avaliado (2002-2006), a comparação entre as três análises mostrou que o MO e a AC superestimaram o número total de VCANs com diferenças de 18 e 17 casos, respectivamente, já o número total de dias de atuação dos sistemas apresentou diferenças correspondentes a 23 e 28 dias, respectivamente. A avaliação mensal mostrou

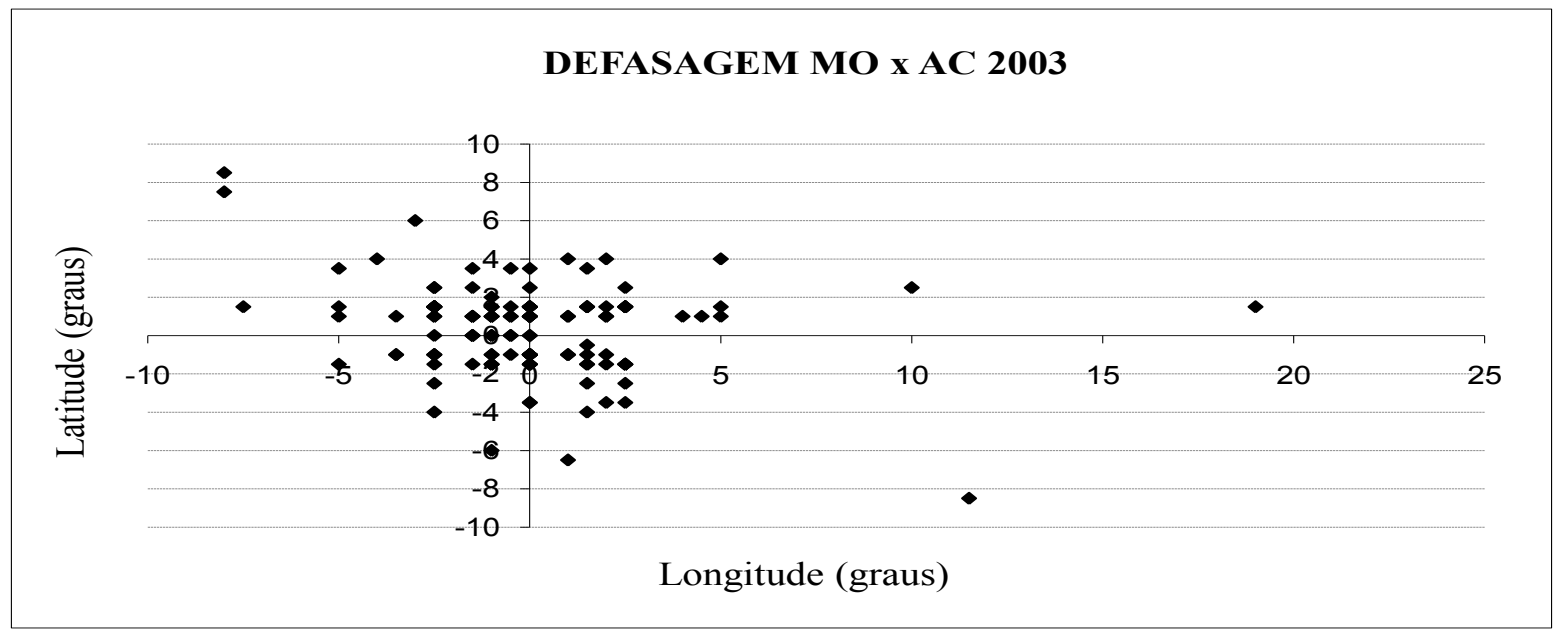

Figura 6 - Como a Figura 3, sendo o MO com relação a AC. 
alguns meses em que a $\mathrm{AC}$ e o $\mathrm{MO}$ subestimaram o número de VCANs e/ou período de vida destes.

Na primeira situação acima, o MO e a AC identificaram sistemas no equador e proximidades que não existiram na IS ou esta não mostrou escoamento com rotação horária durante as animações, e portanto o número de VCANs foi superior. Nos casos em que o $\mathrm{MO}$ e a $\mathrm{AC}$ indicaram um número inferior, o sistema apresentou-se bem configurado na IS, porém na AC não foi observada a configuração fechada, já no MO alguma condição não foi satisfeita. Estas justificativas foram usadas nas discussões sobre o número de dias com atuação desses sistemas.

A avaliação da distribuição espacial dos sistemas obtida pelo $\mathrm{MO}$, durante a validação, mostrou boa representação de algumas características padrões dos sistemas, como direção do deslocamento e região de formação. A formação da maioria dos vórtices ocorreu na área que abrange a costa do NEB e Oceano Atlântico Sul adjacente, compreendendo a faixa latitudinal de $0^{\circ}$ a $20^{\circ} \mathrm{S}$ e nas áreas de maior ocorrência de formação, houve concentração desses sistemas, indicando que muitos casos têm um lento deslocamento.

Conforme visto, o MO conseguiu reproduzir satisfatoriamente algumas das principais características (número de ocorrência, número de dias com atuação, posição, direção do deslocamento e região de formação) associadas aos vórtices que atuam na região Tropical Sul. Isto sugere aplicação do mesmo em centros operacionais de tempo como o CPTEC/INPE.

\section{AGRADECIMENTOS}

Os autores agradecem ao Conselho Nacional de Desenvolvimento Cientifico e Tecnológico (CNPq) pelo auxílio financeiro concedido, à Divisão de Satélites Ambientais (DAS) do INPE e ao Dr. Jorge Conrado Conforte do CPTEC/INPE pelos dados fornecidos, e aos avaliadores anônimos deste artigo que contribuíram valiosamente com sugestões e correções.

\section{REFERÊNCIAS BIBLIOGRÁFICAS}

BENGTSSON,L.;KANAMITSU,M.;KALLBERG,P.;UPPALA, S. FGGE research activities at ECMWF. Bulletin of the American Meteorological Society, v. 63, p. 277-303, 1982. CAMPETELLA, C.; POSSIA, N. Upper-level cut-off lows in southern South America. Meteorology and Atmospheric Physics, v. 96, p. 181-191, 2006.

FRANK, N.L. On the energetic of cold lows. Proceedings of the Synposium on Tropicao Meteorology. American Meteorological Society, EIV-I, EIV-6, June 1970.

FUENZALIDA, H.; SÁNCHEZ, R.; GARREAUD, R. A climatology of cutoff lows in the Southern Hemisphere. J. Geophys. Res., v. 10, p. 1-10, 2005.
GAN, M. A. Um estudo observacional sobre as baixas frias da alta troposfera, nas latitudes subtropicais do Atlântico sul e leste do Brasil. 80p. (INPE-2685-TDL/126). Dissertação (Mestrado em Meteorologia) - Instituto Nacional de Pesquisas Espaciais (INPE), São José dos Campos, 1983.

GAN, M. A; KOUSKY, V. E: Vórtices ciclônicos da alta troposfera no Oceano Atlântico Sul. Revista Brasileira de Meteorologia, v. 1, p. 19-28, 1986.

HODGES, K. I. A general Method for tracking analysis and its application to meteorological data. Mon. Wea. Rev., v. 122, p. 2573-2585, 1994.

HOSKINS, B. J.; HODGES, K. I. A new perspective on Southern Hemisphere Storm Tracks. J. Climate, v. 18, p. 4108-4129, May. 2005.

KALNAY, E., M. KANAMITSU, ET AL. The NCEPNCAR 40-Year Reanalysis Project. Bulletin of the American Meteorological Society, v. 77, p. 437-471, 1996.

KOUSKY, V. E.; GAN, M. A. Upper tropospheric cyclonic vortices in the subtropical South Atlantic. Tellus, v. 33, p. 538-551, 1981.

LOURENÇO, M. C. M. Vórtices ciclônicos em altos níveis que atuam no sul da América do Sul. 1996. 77p. (INPE10462-TDI/929). Dissertação (Mestrado em Meteorologia) - Instituto Nacional de Pesquisas Espaciais (INPE), São José dos Campos, 1996.

MISHRA, S. K.; RAO, V. B.; GAN, M. A. Structure and evolution of the large-scale flow and an embedded upper tropospheric cyclonic vortex over northeast Brazil. Mon. Wea. Rev., v. 129, p. 1673-1688, 2001.

PALMÉN, E.; NEWTON, C.W. Three dimensional flow patterns in extratropical disturbances. In:____ Atmospheric circulations systems, New York, Academic Press, 1969. Cap. 10, p. 274-278.

RAMIREZ, M. C. V. Padrões climáticos dos vórtices ciclônicos em altos níveis no Nordeste do Brasil. 1996. 132p. (INPE-6408-TDI/618). Dissertação (Mestrado em Meteorologia) - Instituto Nacional de Pesquisas Espaciais (INPE), São José dos Campos, 1997.

SILVA, L. A influência do vórtice ciclônico de altos níveis (VCANs) sobre a precipitação do Nordeste do Brasil (NEB) e as características associadas. 109p. Dissertação (Mestrado em Meteorologia) - Instituto Nacional de Pesquisas Espaciais (INPE), São José dos Campos, 2005.

SIMPSON, R. H. Evolution of the Kona Storm, a subtropical cyclone. Journal of Meteorology. v. 9, p. 24-35, 1951.

SINCLAIR, M. R. An objective cyclone climatology for the Southern Hemisphere. Mon. Wea. Rev., v. 122, 2239-2256, 1994. 